duct." The patient, a woman thirty.two years of age, experienced attacks of swelling of the right parotid gland, recurring every five or six months between the summers of 1875 and 1877 . The swelling always appeared somewhat suddenly during meals, and was attended with a feeling of discomfort but no actral pain; it lasted for about half an hour and then rapidly subsided. In the summer of 1878 an attack of more considerable swelling occurred, of longer duration and attended with much pain, especially during meals. The swelling subsided after the escape of some drops of thick pus from Stensen's duct. The attacks now recurred more frequently, sometimes after intervals of not more than two or three weeks, and on one occasion the swelling lasted eight days. No treatment beyond the application of dry warmth to the cheek was adopted. Dr. Krieger, who treated the patient, found that the masses which escaped from the duct consisted of pus and fine filamentous cylinders, the fibrinous nature of which was demonstrated microscopically by Dr. Friedländer. The patient was taught to pass a fine platinum wire sound along the duct and thus obtained relief, for on withdrawing it thick fibrino-purulent matter escaped and after this a free flow of saliva. At the beginning of November, 1878, another attack occurred in which not only the right gland, but to a much slighter degree the left, was affected. After the patient's midday meal Kussmaul found great swelling in front of and below the right ear, the skin was red and hot, and pressure was painful. The sound could be passed only a few millimetres along the duct, but on withdrawing it some yellow, creamy pus escaped, followed by a filamentous thread and clear saliva, after which the sound could be introduced for four centi. metres. In an attack in January, 1879, the thread which escaped was three centimetres long and as thick as a knitting needle. This was examined by ron Recklinghausen, who found that it consisted of a fine membranous deposit composed of a network of fibrin containing pus and epithelial cells. The patient was seen some time subsequently and had had no further attacks. A chlorate of potash lozenge was taken daily. Another case which is worthy of notice was published by Berthold stiller of Budapest. ${ }^{3}$ A man aged thirty-six hurriedly summoned Dr. Stiller on account of a rapidly increasing swelling of the right cheek which appeared during dinner. The swelling corresponded with the right parotid gland and was the seat of a sensation of warmth and discomfort but not of actual pain. Nothing unnatural could be seen in the mouth. Dry heat was applied to the cheek but did no good, for on the following day the swelling was larger and painful. Whilst the patient was handling and squeezing the swelling he spat out it shreddy purulent mass, whereupon the mouth filled with a clear watery fluid. The swelling rapidly diminished and on the following day had disappeared. Subsequently the patient experienced milder attacks every three to six months, and the swelling was invariably relieved by pressure applied to the gland. These two cases afford strong support to the view that I had independently formed as to the nature of at least the first three of those which I have recorded. In koth the rapid subsidence of the swelling after relief of the obstruction in the duct shows that it was due simply to retention of secretion and not to inflammation of the gland. In my cases, on the other hand, the persistence of the swelling and induration would point to the occurrence of inflammation of the gland lobules of a kind comparable to that which may occur under similar circumstances in other secreting glands, notably the breast. In Cases 4 and 5 evidence that the primary mischief was in Stensen's rluct is wanting, but I strongly su=pect that these were also of the same nature.

As perhaps throwing a side light on the subject it may be mentioned that two cases of recurrent swelling of both parotid glands in association with xerostomia or "drymouth" have recently been recorded. In Battle's case both parotids had alternately increased and diminished in size for a period of two years, and whilst the patient was under observation and the glands were at their minimum size the smaller was as large as a walnut, and both were firm and lobulated. In Chappell's case " the parotid glands "would suddenly enlarge at times, bccome tender, and after two or three days gradually subside." Whilst the patient was first under observation, in May, 1894, "both parotids

s Wiener Medicinische Wochenecbrift, 1881, No. 19.

Transactions of the Clinical Society, 1895, p. 282 .
5 New York Medical Journal, Feb. 29th, $18 \subseteq 6$. were much enlarged, especially the right, and seemingly consisted of three or four nodular masses which were harc and non-sensitive and resembled those of a patient with mumps." In February, 1895, the glands had increastd considerably in size. It may be considered probable that in these cases an inflammation of Stensen's duct, associated with the unhealthy condition of the mouth, was the cause of the parotid swellings. A condition similar to that under consideration has been met with in the submaxillary gland by Ipscher. ${ }^{\circ}$ He was burriedly summoned to a man aged thirty on account of a swelling which had suddenly formed in the right submaxillary region. Before Dr. Ipscher arrived the patient had relieved himself by pressing on the swelling when "a small white thread" appeared in the mouth followed by "a quantity of sour-tasting water." The right submaxillary gland was still considerably swollen, and the corresponding part of the floor of the mouth was reddened. This was the third attack, and in each of the two previous ones pressure on the swelling had been followed by a similar result. This case was published as the result of Kussmaul's communication, and was regarded by Ipscher as of the same nature. The only instance of swelling of the submaxillary gland, possibly belonging to this class of cases, which I have observed was that of a boy eight years of age who was brought to the Victoria Hospital on Feb. 9th, 1894. The swelling in the neck formed during the night in the winter of 1892 and did not subside for eight months. In November, 1893, it reappeared, and when I saw the boy the swelling evidently involved the right submaxillary salivary gland. There was nothing abnormal in the appearance of Wharton's duct, and a probe could easily be passed along it. Three weeks later the gland was smaller. The nature of this case was obviously doubtful.

I have thought these cases worthy of being recorded, although necessarily incomplete in many respects, because they illustrate a condition of some importance, if only on account of its superficial resemblance to mumps. It is a useful rule of practice always to examine the duct of a swollen salivary gland. If there is any probability that the swelling results from blockage of the duct by inflammatory products the regular passage of a fine probe may be expected to give relief, but induration of the gland is likely to persist for a considerable period.

Wermouth-street, $\mathbf{w}$.

\section{SOME CASES OF VENTROFIXATION OF THE UTERUS.}

\section{Br HASTINGS GILFORD, F.R.C.S. ExG.}

I rentuRe to bring forward these few cases because the discussion on the subject of ventrofixation of the uterus by the British Gynæcological Society gives them some value from a statistical point of view. But apart from this they are, I think, worthy of publication owing to certain special features of interest. Thus, in one case the operation. was done because of the perforation of the uterus by a curette. In another intussusception of the bowel set in aiter the operation, resulting in self-cure. In two of them both ovaries were also removed. In four Keith's operation was performed.

CASE 1.-A woman aged thirty-fice years had severe prolapse of the uterus for five years and had suffered much from enlarged and painfal ovaries. No pessary could be tolerated and she became thin, haggard, and utterly dispirited from constant pain and uneasiness. Both oriries were removed in June, 1893, and the uterus was sewn to the edges of the wound with four silk sutures. It was nearly eighteen months before she had fully recovered her health, but now she is in an excellent condition.

C.ASN 2.-This patient was a woman aged thirty years. Keith's operation was advised owing to persistent pain in the right ovary and retroflexion of the uterus, but the left orary keing found unhealthy at the time of operation was also removed. This was done on April 18th, 1895, and so far as the displacement is concerned the result is satisfactory, but the ovarian disturbance, though better, has not yet disappeared.

CAsk 3.-In November, 1894, I was asked to see a woman 
aged twenty-six years with a view to the operative treatment of a band which could be felt in the upper part of the ragina, apparently dragging the cervix towards the right and tiling the ute: us to the lett. The uterus was also displaced backwards and the left ovary was large, prolapsed, and excessively tender. The patient herself was anæmic and depressed by two years of ovarian disturbance which had resisted all treatment. The band was first cautiously divided without influencing the displacement, and then Feith's operation was performed by clamping the left ovary externally. Convalescence was retarded by the appearance of white leg on the left side on the fourteenth day. She is now, however, in good health.

CASE 4.-This patient had suffered from pelvic trouble for sig years and had been treated by various gynæcologists wilhout permanent benefit. In the beginning of 1892, when I first saw her, her husband was abroad. Her symptoms were then not of sufficient severity to render an operation necessary, but in the summer of 1894 her husband returned and, her symptoms becoming progressively worse, it was deciced that the case should be taken in hand. I found, on examining under ether, that the right ovary was enlarged and so displaced as to bring it under the fundus of the uterus, which was bent down over it. After six weeks' futile treatment by rest in bed, hot-water irrigations, and pessaries I removed the ovary by clamping the pedicle in the wound. She made a speedy recovery and is now quite well.

CASE 5.-In January, 1893, I was consulted by a woman aged twenty-nine years for pelvic pain and metror rhagia. I found she had an enlarged and retroflexed uterus from which there had been a discharge of blood for five weeks, following amenorrhcea of six weeks' duration. She was given an anæ:thetic, and after I had removed the products of conception the uterus was carefully curetted with a blunted "sharp" curette and irrigated with boiled water. It was then noticed that less water came out than flowed in, and a sound was passed which, to my horror, went in up to the bandle without a hitch. It was now evident that the fundus of the uterus had been perforated by the curette, and on taking into consideration the size of the aperture, the fact that the discharge from the vagina was offensive, and that some of the contents of the uterus had been washed into the abdominal cavity, it seemed best to perform an abdominal section. The necessary instruments were therefore sent for and prepared while the ansesthetic was continued. On opening the abdomen and inspecting the uterus it was found that an irregularly starshaped hole had been made through the highest part of the fundus. It was quite closed, and the edges, though ragged, were neither soft nor bruised. After washing out the abdominal cavity with boiled water the fundus of the uterus was sewn to the anterior abdominal wall in such a way that the part adjacent to the perforation was extra-peritoneal. Catgut sutures were used to attach the superficial layer of the uterus to the peritoneum of the abdominal wall, while two silk sutures transfixed the parietes and went deep into the substance of the uterus, one above the aperture and the other below it. A little bloody discharge afterwards oozed from the fundus of the uterus, but in fourteen days the wound had closed and the uterus now remains firmly fixed in place. That which most surprised me in this case was not so much the complete absence at the time of any indications of the mischief I had done with the curette, though I had it well under control, as the lack of that softness or cedema of the uterine wall which one is led to suppose is usually present in these cases. The tissue of the uterus on inspection vas seemingly of normal consistency. Moreover, the curette was apparently pushed and not scraped through the uterus, for tise perforation was not at the sides where the scraping action of the instrument would come into play, but at the fundus where such an action would have been at a minimum. Writers (e g., Mr. Lawson Tait ${ }^{1}$ ) have often called attention to the frequency of this accident, of the impossibility of always avulaing it, and of the rarity with which it is attended with evil results. But under the circumstances I have detailed I think it must be safer to deal with the uterus in the way $I$ have indicated than to leave the matter to chance. The cure of the displacement is also of positive advantage to the patient. I do not think there can be any question that such a course is preferable to that of removal of the uterus, which has been suggested by some continental operators.

CASE 6.-This was of a similar nature to the preceding, except that the symptoms were more severe and the displacement had resisted all treatment. At the time the patient was placed under my care the bleeding had be $\in \mathrm{b}$ going on for nearly three months and the discharge was offen=ive. After curetting the uterus Keith's operation was performed, the right ovary being clamped. On the second day, after the subsidence of the nausea due to the ether, she complained of griping pain across the middle of the abctomen attended with nausea and occasional eructations and vomiting. The pulse was 98. Sulphate of magnesium and enemata of soap and water, of turpentine, and of glycerine (4 cz.) were successively tried without result. Nothing but hot water was now given by the mouth, and after twelre hours the same aperients were tried aqain with good effect. The pinching pain and nausea and vomiting still continued at increasing intervals for nearly a month after this date, and the bowels acted loosely from two to six times a day. The temperature also went up each erening to $102^{\circ} \mathrm{F}$, and dropped to the normal point in the morning. The abdomen became slightly tender and dis= tended, but there were no local signs indicative of pelvic or abdominal mischief. On the fifth day the patient had a shivering attack, when the temperature rose to $102.5^{\circ}$. The uneasiness with which one regarded the continuance of these symptoms was, howerer, much lessened by the fact that the pulse was of good volume and not rapid, the tongue was moist, and the patient herself looked well and was free from the feeling of illness. On the sixth day the motions became exceedingly offensive. On the fourteenth day the mystery was partly cleared up by the protusion from the anus of $\%$ shred of membrane which stank horribly, and proved on inspection to be a piece of the entire thickness of the large intestine, triangular in shape, the base being six inches and the sides five inches long. No other pieces were found. Other symptoms continued, however, with diminishing severity for a month after the operation. Though never before so affected, she now suffered from mixed internal and external hæmorrhoids A rectal examination revealed notbing amiss. The patient is now, six weeks after the operation, steadily improving, and has lost all her symptoms save slight looseness of the bowels. For lack of any better explanation I attribute the complication that ensued in this case to intussusception of the colon from irregular contraction of its walls during or subsequently to etherisation. The case is a good example of the unforeseen accidents that are prone to attend all major operations. It serves to show that no abdo. minal section can be considered safe however simple the operation.

CAsE 7.-This case was similar to Case 3. The ratient was twenty-six years old and the results have been excellent.

In none of these instances has the uterus become again displaced. In none of them has there been any trouble with the bladder with the exception of frequent micturition for a few hours after the operation ; and even that was present in only two cases. Pregnancy has not followed in any case. I prefer Keith's operation even when there is no ovarian disease because of the mortality which has hitherto attended the performance of immediate suturing of the uterus to the abdominal wall. This mortality $I$ feel sure is far bigher than may be inferred from published statistics, for I have myself heard of several fatal cases which have never been made public.

Reacling.

\section{ON THE DISSECTION TO EXPOSE THE LENTICULAR GANGLION.}

BY FDWARD FAWCETT, M.B. EDIN.

PROTESSOR OF ANATOMY, CAIVERSITY COLLEGE, LRISTOX.

THIS is a ganglion which is often read about but seldom seen-at all events, satisfactorily. The object of this short communication is to point out how not only the ganglion but its minutc branches and roots may always be demonstrated with no difficulty whatever. Most works on practical anatomy recommend the student to dissect the orbit from above. So far as the general contents of the orbital cavity are concerned this method is as economical and satisfactory, perhaps, as any, but it is extremely unsatisfactory so far as exposure of the lenticular ganglion is concerned. Professor 